International Journal of Engineering \& Technology, $8(4)(2019) 398-401$
International Journal of Engineering \& Technology
SPC
Website: www.sciencepubco.com/index.php/IJET
Research paper

\title{
Performance analysis of highly improved hybrid turbo codes for 4G wireless networks
}

\author{
M. Jose Raj $^{1}{ }^{*}$, Dr.Sharmini Enoch ${ }^{2}$ \\ ${ }^{I}$ Research Scholar Dept of ECE, Noorul Islam Center for Higher Education Kanyakumari District, Tamilnadu, India \\ ${ }^{2}$ Professor Dept of ECE, Noorul Islam Center for Higher Education Kanyakumari District, Tamilnadu, India \\ *Corresponding author E-mail:
}

\begin{abstract}
Efficient error correcting codes are essential in modern digital communication systems. Highly Improved Hybrid Turbo Code (HIHTC) is a low complex error and efficient error correcting code with excellentBit Error Rate (BER) which is comparable to Low Complexity Hybrid Turbo Codes (LCHTC), Improved Low Complexity Hybrid Turbo Codes (ILCHTC) and other Hybrid Turbo Codes. Rate 1/3 HIHTC shows a BER of 10-5 for E b/No of $1.7 \mathrm{~dB}$ which is closer to the E b/No of Improved Low Complexity Hybrid Turbo Codes. In this paper we analyze the performance of HIHTC in comparison with otherLow Complexity Hybrid Turbo Codes, for their performance in $4 \mathrm{G}$ and $5 \mathrm{G}$ wireless networks
\end{abstract}

Keywords:Zigzag-Hadamard (ZH); A Posteriori Probability (APP); Bit Error Rate (BER); Decoder Complexity(DC); Hybrid Turbo Code (HTC).

\section{Introduction}

Shannon's limit gives the relationship between Signal to Noise Ratio and Channel Capacity of a communication channel. A reliable and efficient error correcting code is essential for error free communication and it should obey the Shannon's limit rule. Since reflection of signals in multiple channels and fading of signals lead to errors the error correcting code should be efficient with low BER. Turbo convolution codes (TCC) [2] is the efficient error correcting code with better BER. But the decoder complexity of TCC is more. The hardware requirement is more and need more complex Trellis decoding process to decode one bit. MAP based algorithmafter puncturing is used to improve the code rate .But the performance is not much improved. HIHTC [1] is the improved version of ILCHTC which is the combination of TCC and ZH codes and hence called Hybrid Low Complexity Hybrid Turbo Code.. In HIHTC Complexity is very much reduced and BER is improved and approaches Shannon's limit. We analyze the performance of Hybrid Low Complexity Hybrid Turbo Code for high speed data rates in $4 \mathrm{G}$ and $5 \mathrm{G}$ wireless networks.

\section{Encoder description}

HIHTC encoder using Zigzag [5] Hadamard code and Recursive Systematic Convolution (RSC) code as shown in Fig.(1)

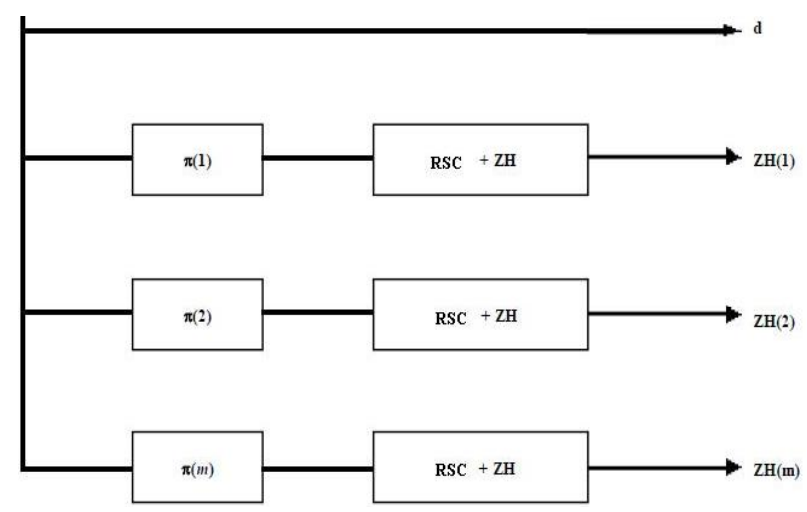

Fig. 1:HIHTC Encoder.

The HIHTC overall encoder has two constituent encoders. The first encoder is the RSC encoder and the second one is the zigzaghadamard encoder. Initially the data is directly given to the encoder, then the data is interleaved using a random interleaver and then encoded by the convolution encoder and the interleaved data is given to the zig-zaghadamard encoder. Finally the overall data is given to the decoders.

In HIHTC the information bits are arranged in a rectangular array of size PxQ

$(p+(j-1) X q)^{\text {th }}$ information bit is denoted by $d(j, k)$

$$
\begin{aligned}
& \mathrm{d}=\{\mathrm{d}(\mathrm{j}, \mathrm{k})\}, 1 \leq \mathrm{j} \leq \mathrm{P} \text { and } 1 \leq \mathrm{k} \leq \mathrm{Q} \\
& \mathrm{ZH}^{\mathrm{m}}(\mathrm{k})=\sum_{\mathrm{j}=1}^{\mathrm{J}} \mathrm{d}(\mathrm{h}, \mathrm{k})+\mathrm{ZH}^{\mathrm{m}}(\mathrm{k}-1) \bmod
\end{aligned}
$$

P- row length of array matrix

Q- Column length of array matrix

d- encoded data bit 
$\mathrm{h}, \mathrm{k}$ - data positions

$\mathrm{m}$ - number of constituent encoders

\subsection{RSC encoder}

The recursive systematic convolutional (RSC) [6] encoder is obtained from the nonrecursive nonsystematic (conventional) convolutional encoder by feeding back one of its encoded outputs to its input as shown in Figure 2. Figure 3 gives the State Diagram of RSC Encoder.

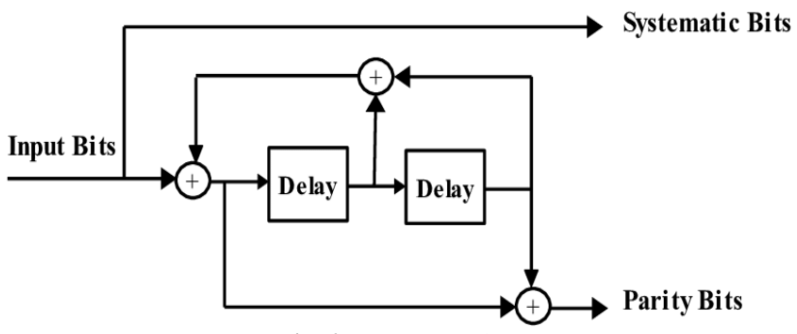

Fig. 2: RSC Encoder.

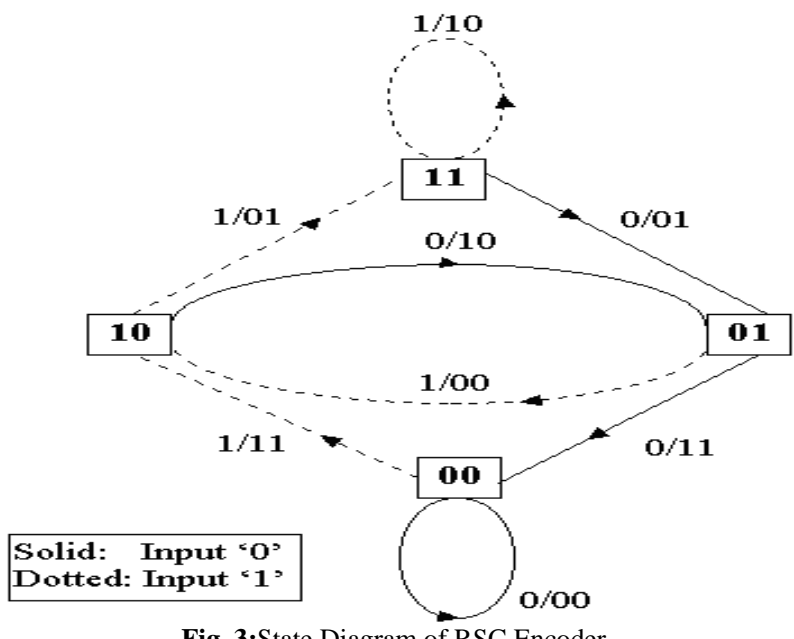

Fig. 3:State Diagram of RSC Encoder.

\subsection{Zig-zag encoder}

Zigzag Hadamard codes arerepresentedby a highly structured zigzag graph with each segment has been specified by a Hadamard codeword. This $\mathrm{ZH}$ codes enjoy extremely simple encoding and very-low-complexity using Soft-input soft-output (SISO) decoding. ZH codes are convolution-like codes and hence can be represented by a two state trellis with four bundles of parallel branches corresponding to the first and the last bit in a Hadamard code segment. The Hadamard code matrix is implemented in Zig zag code .Hence the complexity of HIHTC is considerably reduced . Figure 4 and 5 gives the Zig Zag Encoding Sequence.

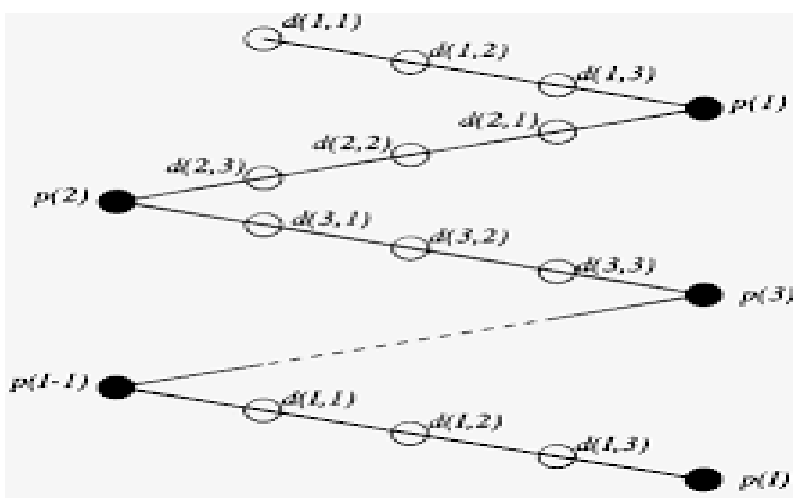

Fig. 4:Zig Zag Encoding Sequence.

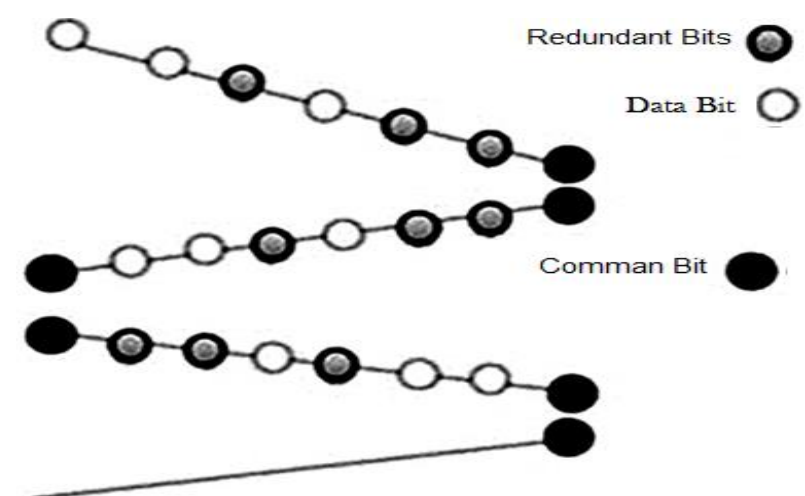

Fig. 5:Zig-Zag Coding with Unpunctured Pattern.

The Hadamard matrix is given in Equation 3

$$
\mathrm{H}_{\mathrm{n}}=\left(\begin{array}{c}
+\frac{\mathrm{h}_{\mathrm{n}}}{2}+\frac{\mathrm{h}_{\mathrm{n}}}{2} \\
+\frac{\mathrm{h}_{\mathrm{n}}}{2}-\frac{\mathrm{h}_{\mathrm{n}}}{2}
\end{array}\right)
$$

\section{Decoder description}

Soft In Soft Out (SISO) A Posteriori Probability (APP) is used for decoding Zigzag Hadamard codes.. The Log Likelihood Ratio (LLR) are calculated for the received bits and then LLRs are arranged in array format $\mathrm{H} \times K$.

The algorithm for decoding process is given below:

i) Each row of the array is decoded using a priori LLRs as input to Convolution decoder. Then each row of the information bits produced Output in the form of a posteriori LLR.

ii) The damping factor, DF, is applied to a posteriori Long likelihood Ratio (LLR) found in steps.

iii) Then each column of the array decoded using, output of as A priori LLRs for Zigzag Hadamarddecoder.

iv) The overall decoder working as a global iterative decoder.

v) The soft information required by the Turbo decoder is obtained though maximum a posteriori (MAP) detection, which consists of evaluating the LLR of the a posteriori probabilities of a bit Equation 4,5,6 gives the decoder LLR.

$$
\begin{gathered}
L(i)=\log \frac{P(C(i)=+1 / x)}{P(C(i)=-1 / x)} \\
=\log \frac{P(X(i)=+1 / x) P(C(i)=+1 / x)}{P(X(i)=-1 / x) P(C(i)=-1 / x)} \\
=\log \frac{\Sigma C \in \pm h^{j}: C(i)-+1 P(\%) P(c)}{\Sigma C \in \pm h^{j}: C(i)=-1 P(\%) P(c)} \\
\mathrm{i}=0.1 \ldots \ldots 2^{r}-1
\end{gathered}
$$

\section{Performance analysis}

Concatenated coding schemes, using relatively simple constituent Convolution and block codes like zig-zag codes, can achieve performance close to the theoretical Shannon's limits . Concatenated zig-zag codes are low complexity parallel concatenated block codes and turbo convolution codes is the efficient error correcting code with better BER. These two codes are combined in concatenated scheme to get Highly Improved Hybrid Turbo Code (HIHTC).If Soft-In Soft-Out (SISO) decoding is implemented, the Rate-1/2 concatenated Zigzag decoder requires 20 Average Equivalent Operations per Information Bit Iteration (AEO/IB/I). . For long length of interleaver the BER of the code increases. However, a long interleaver length leads to more time delay as each decoder requires longer latency in interleaving and deinterleaving the received bits. The simulation results show that BER performance of HIHTC is better than that of Improved Low Complexity Hybrid Turbo Codes and Low Complexity Hybrid Turbo Codes. The decoder complexity of HIHTC also very less than the 
decoder of ILCHTC and LCHTC [8]. The HIHTC achieves excellent BER performance as well. For a BER of $10^{-5}$, the $\mathrm{E} b / \mathrm{N}_{o}$ is $1.7 \mathrm{~dB}$ which is $0.1 \mathrm{~dB}$ more than that of TCC. Therefore, HIHTC is highly suitable for $4 \mathrm{G}$ and $5 \mathrm{G}$ wireless communication applications. The Figure 6 gives the Performance of HIHTC, ILCHTC and LCHTC,TCC

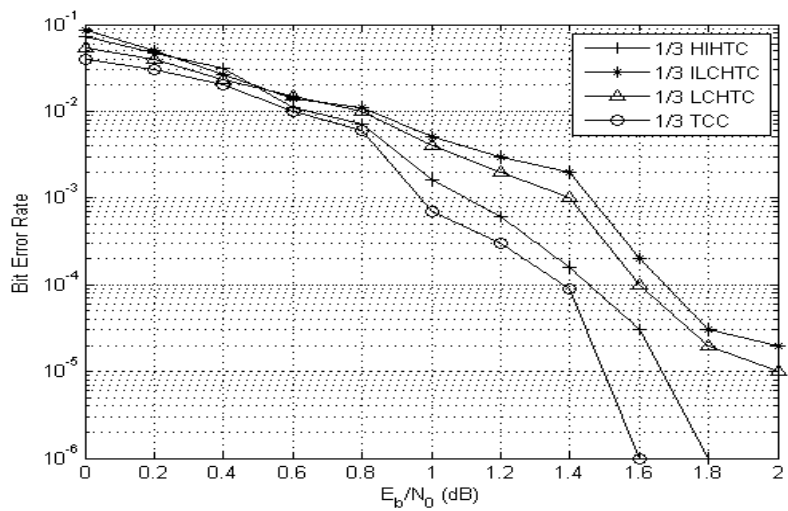

Fig. 6:Performance of HIHTC, ILCHTC and LCHTC,TCC.

The HIHTC with different code rate are analyzed. Different code rates such as $2 / 3,1 / 2,1 / 3$ are analyzed with HIHTC. Increase is redundant bits shows better performance. The rate $1 / 3$ HIHTC shows very good performance than rate $1 / 2$ and $2 / 3$. Figure 7 gives Performance of HIHTC for Different Code Rates.

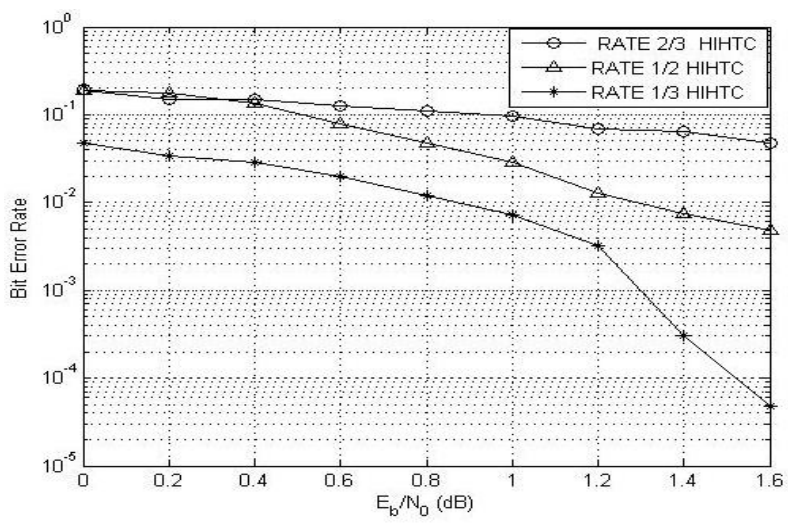

Fig. 7:Performance of HIHTC for Different Code Rates.

\section{Complexity analysis}

The main objective of HIHTC is to reduce the decoder complexity and make it suitable for modern wireless communications. Let $\mathrm{Q}_{\mathrm{A}}$ be the number of additions / Information Bit/ Iteration (A/ IB /I) and $\mathrm{Q}_{\mathrm{M}}$ be the number of multiplications / Information Bit/ Iteration required to decode one bit.Information Bit/ Iteration required to decode one bit. QM, QA gives the number of multiplication and addition.

Table 1 gives Complexity Comparison Chart for HIHTC, ILCHTC and LCHTC,TCC

For a HIHTC decoder

$\mathrm{Q}_{\mathrm{M}}=[\mathrm{L} *(8 * \mathrm{~S}-2)] / \mathrm{H}$

$\mathrm{Q}_{\mathrm{A}}=\{[(16 * \mathrm{~S}-1) / \mathrm{H}]+[(3+2) / \mathrm{H}]\}-1$

Table 1: Complexity Comparison Chart for HIHTC, ILCHTC and LCHTC,TCC

\begin{tabular}{lllll}
\hline Decoder & $\mathrm{R}$ & Parameter & Qm & Qa \\
\hline \multirow{2}{*}{ TCC } & $1 / 2$ & $\mathrm{M}=2$ & 120 & 256 \\
& $1 / 3$ & $\mathrm{M}=2$ & 120 & 256 \\
LCHTC & $1 / 2$ & $\mathrm{M}=2 . \mathrm{H}=3 . \mathrm{L}=2$ & 40 & 96 \\
& $1 / 3$ & $\mathrm{M}=2 . \mathrm{H}=3 . \mathrm{L}=2$ & 60 & 145 \\
\multirow{2}{*}{ ILCHTC } & $1 / 2$ & $\mathrm{M}=2 . \mathrm{H}=3 . \mathrm{L}=2$ & 30 & 79 \\
& $1 / 3$ & $\mathrm{M}=2 . \mathrm{H}=3 . \mathrm{L}=2$ & 60 & 150 \\
\multirow{2}{*}{ HIHTC } & $1 / 2$ & $\mathrm{M}=2 . \mathrm{H}=3 . \mathrm{L}=2$ & 25 & 60 \\
& $1 / 3$ & $\mathrm{M}=2 . \mathrm{H}=3 . \mathrm{L}=2$ & 50 & 140 \\
\hline
\end{tabular}

The HIHTC shows only 50 multiplications and 140 additions to retrieve one bit. In case of TCC multiplications needed is 120 and additions needed is 256. In ILCHTC multiplications needed is 60 and additions needed is 150 . Overall the complexity of HIHTC is less as compared to other codes

\section{Channel performance}

The performance of HIHTC is simulated for Noisy and fading channels. The HIHTC shows better results as compared to ILCHTC, LCHTC and TCC. For fading channel also the performance ofHIHTC[1] is superior. Figure 8 gives Performance of Codes for AWGN Channel.

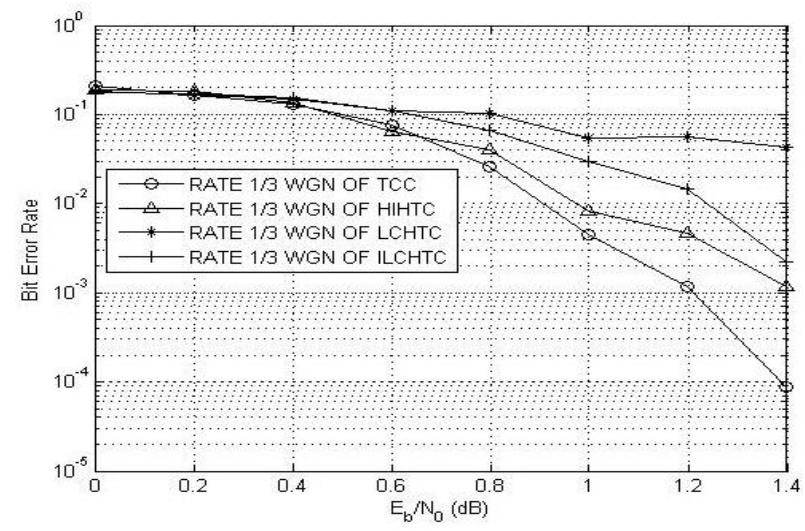

Fig. 8:Performance of Codes forAWGN Channel.

For path 3 fading channel the HIHTC shows BER $10^{-4}$ for the E $\mathrm{b} / \mathrm{N}_{\mathrm{o}}$ of $1.58 \mathrm{~dB}$. For path 5 shows BER $10^{-3}$ for the $\mathrm{E} b / \mathrm{N}_{\mathrm{o}}$ of 1.4 $\mathrm{dB}$ in fading channel. Ultimately the HIHTC gives reliable performance in noisy and fading channel. Figure 9 gives Performance of codes for Raleigh Channel.

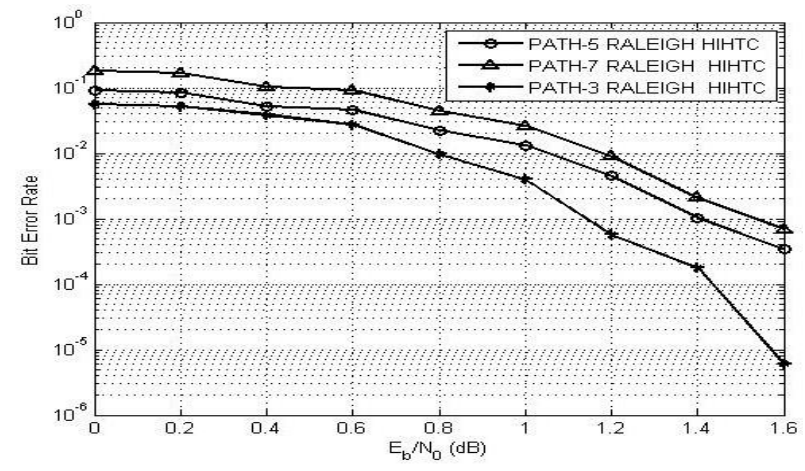

Fig. 9:Performance of Codes for Raleigh Channel.

\section{Performance in OFDM}

OFDM has been adopted in the wireless arena where the standards like 802.11a, 802.11n, 802.11ac and more. It has also been chosen for the cellular telecommunication's standard LTE / LTE-A, and in addition to this it has been adopted by other standards such as WiMAX and many more. The HIHTC is suitable for very high speed applications such as UMTS, 4G, 5G, Mobile WIMAX etc. HIHTC shows BER $10^{-4}$ for the $E$ b/ $\mathrm{N}_{0}$ of $14.5 \mathrm{~dB}$. In case of ILCHTC for $\mathrm{E}_{\mathrm{b}} / \mathrm{N}_{\mathrm{o}}$ of $15 \mathrm{~dB}$ the BER is $10^{-3}$. Hence it is clear that HIHTC can support error free communication in the above networks. Figure 10 gives Performance of HIHTC, ILCHTC, TCC for OFDMA 


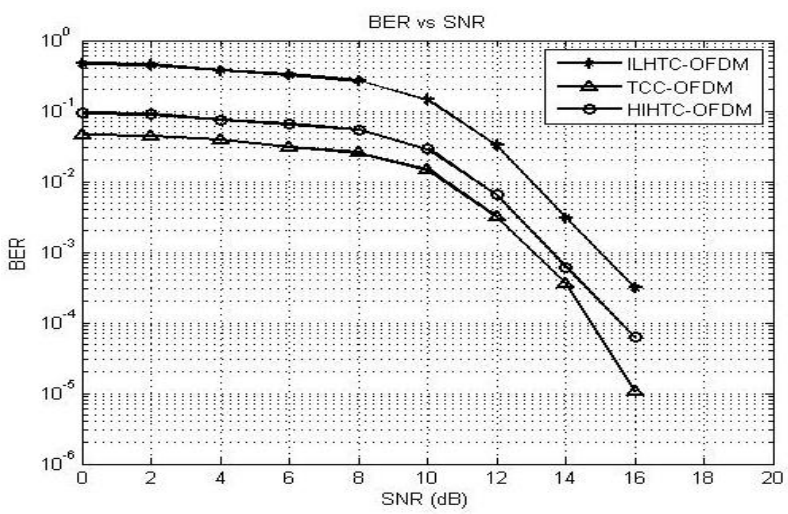

Fig. 7: Performance of HIHTC, ILCHTC,TCC for OFDMA.

\section{Conclusion}

The performance of HIHTC is analyzed for various noisy and fading channels also for OFDMA access technique. The HIHTC showsBER $10^{-4}$ for the $\mathrm{E} b / \mathrm{N}_{\mathrm{o}}$ of $14.5 \mathrm{~dB}$.which superior performance with OFDMA which is suitable for $4 \mathrm{G}$ and $5 \mathrm{G}$ wireless applications. The complexity also very much reduced to make it suitable for various modern communication systems. The complexity of HIHTC decoder is $45 \%$ less than the High complex TCC decoder.

\section{References}

[1] M. Jose Raj and Dr. Sharmini Enoch, "Performance Analysis of Highly Improved Hybrid Turbo Codes.

[2] C. Berrou, A Glavieux, and P. Thitimajshima, "Near Shannon limit error correcting coding and decoding turbo-codes," in Proc. 1993 IEEE Int.Conf. Commun., May 1993, pp. 1064-1070.

[3] S. Benedetto and G. Montorsi, "Unveiling turbo codes: some results on parallel concatenated coding schemes," IEEE Trans. Inf. Theory, vol. 42, pp. 409-428, Mar. 1996. https://doi.org/10.1109/18.485713.

[4] M. C. Valenti and J. Sun, "The UMTS turbo code and an efficient decoder implementation suitable for software defined radios," International J.Wireless Inf. Netw., vol. 8, no. 4, pp. 203-214, Oct. 2001.

[5] L. Ping, X. Huang, and N. Phamdo, "Zigzag codes and concatenated zigzag codes," IEEE Trans. Inf. Theory, vol. 47, no. 2, pp. 800807, Feb.2001.https://doi.org/10.1109/18.910590.

[6] L. Ping, "Turbo-SPC codes," IEEE Trans. Commun., vol. 49, no. 5, pp.754-759, May 2001.https://doi.org/10.1109/26.923796.

[7] K. Wu and L. Ping, "An improved two-state turbo-SPC code for wireless communication systems," IEEE Trans. Commun., vol. 52, no. 8, pp. 1238-1241, Aug. 2004.https://doi.org/10.1109/TCOMM.2004.833042.

[8] A. Bhise and P. D. Vyavahare, "Low complexity hybrid turbo codes," in Proc. IEEE Wireless Commun. Netw. Conf., Las Vegas, pp. 1525-1535, Mar. 2008.https://doi.org/10.1109/WCNC.2008.190.

[9] Lu, P. Y., Lu, E. H., I\& Chen, T. C. (2014). An efficient hybrid decoder for block turbo codes. IEEE Communications Letters, 18(12) 2077-2080.https://doi.org/10.1109/LCOMM.2014.2364229.

[10] Suman, SumitDalal, A Novel Approach for Designing Hybrid Turbo Codes Using Double Stage Interleaver, International Journal of Engineering Sciences $\backslash \&$ Research Technology, ISSN: 2277-9655. 